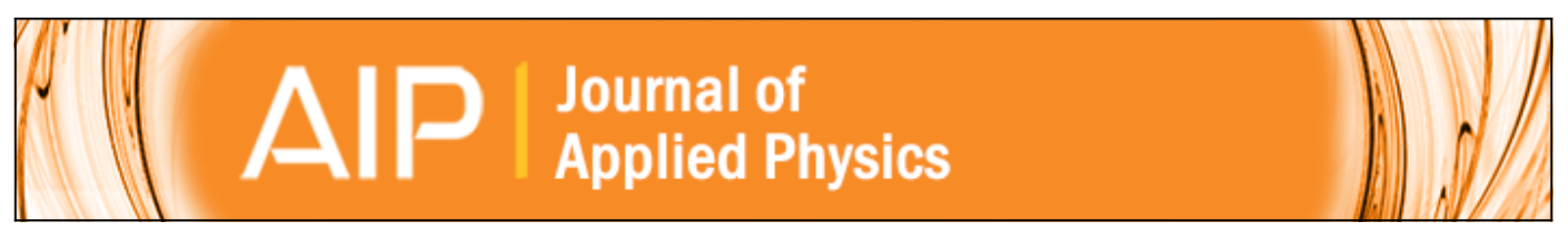

Avalanche-like vortex penetration driven by pulsed microwave fields in an epitaxial LaSrCuO thin film

P.-de-J. Cuadra-Solís, J. M. Hernandez, A. García-Santiago, J. Tejada, J. Vanacken, and V. V. Moshchalkov

Citation: Journal of Applied Physics 114, 233902 (2013); doi: 10.1063/1.4848997

View online: http://dx.doi.org/10.1063/1.4848997

View Table of Contents: http://scitation.aip.org/content/aip/journal/jap/114/23?ver=pdfcov

Published by the AIP Publishing

AlP 


\title{
Avalanche-like vortex penetration driven by pulsed microwave fields in an epitaxial LaSrCuO thin film
}

\author{
P.-de-J. Cuadra-Solís, ${ }^{1,2}$ J. M. Hernandez, ${ }^{1,2}$ A. García-Santiago, ${ }^{1,2, a)}$ J. Tejada, ${ }^{1,2}$ \\ J. Vanacken, ${ }^{3}$ and V. V. Moshchalkov ${ }^{3}$ \\ ${ }^{1}$ Grup de Magnetisme, Departament de Física Fonamental, Facultat de Física, Universitat de Barcelona, \\ c. Martí i Franquès 1, planta 4, edifici nou, E-08028 Barcelona, Spain \\ ${ }^{2}$ Institut de Nanociència i Nanotecnologia IN2UB, Universitat de Barcelona, c. Martí i Franquès 1, \\ planta 3, edifici nou, E-08028 Barcelona, Spain \\ ${ }^{3}$ INPAC-Institute for Nanoscale Physics and Chemistry, Katholieke Universiteit Leuven, \\ Celestijnenlaan 200D, B-3001 Leuven, Belgium
}

(Received 22 July 2013; accepted 1 December 2013; published online 16 December 2013)

\begin{abstract}
Different vortex penetration regimes have been registered in the output voltage signal of a magnetometer when single microwave pulses are applied to an epitaxial overdoped $\mathrm{La}_{2-x} \mathrm{Sr}_{x} \mathrm{CuO}_{4}$ thin film in a perpendicular dc magnetic field. The onset of a significant variation in the sample magnetization which exists below threshold values of temperature, dc magnetic field, and pulse duration is interpreted as an avalanche-type flux penetration. The microwave contribution to the background electric field suggests that the nucleation of this fast vortex motion is of electric origin, which also guarantees the occurrence of vortex instabilities under adiabatic conditions via the enhancement of the flux flow resistivity. Flux creep phenomena and heat transfer effects act as stabilizing factors against the microwave-pulse-induced fast flux diffusion. (C) 2013 AIP Publishing LLC. [http://dx.doi.org/10.1063/1.4848997]
\end{abstract}

\section{INTRODUCTION}

The magnetic properties of superconductors are explained in an effective approach to flux static and dynamics in materials with a high density of pinning centers suggested by Bean. ${ }^{1}$ This model assumes that the current density equals its maximum attainable non-dissipative value, the so-called critical current density, anywhere through the current carrying region so that the spatial variation of the flux density decreases linearly with the distance from the surface into the superconductor. Due to the balance between vortex density gradients which drive vortices into the sample and pinning forces which hamper their entry, a metastable critical state is created. This nonuniform flux distribution becomes unstable under certain conditions so that a thermomagnetic instability (TMI) or a vortex avalanche may arise. ${ }^{2}$ This process could be initiated by a small fluctuation of the external magnetic field or the temperature, resulting in a vortex redistribution towards equilibrium, the increase of the sample temperature, and the decrease or quenching of the screening current. The heat released by the moving vortices leads in turn to further flux motion giving as a consequence a positive feedback process. ${ }^{2-4}$ The effect of the vortex and temperature redistribution depends on the ratio $\tau$ of the magnetic flux $\left(t_{\mathrm{mag}}\right)$ and thermal $\left(t_{\mathrm{th}}\right)$ diffusion time constants: ${ }^{2}$ $\tau \equiv t_{\mathrm{mag}} / t_{\mathrm{th}}=\mu_{0} \kappa / C \rho_{f}$. Here $\mu_{0}$ is the magnetic permeability of vacuum, and $\kappa, C$, and $\rho_{f}$ are, respectively, the thermal conductivity, the heat capacity, and the flux flow resistivity of the material. For $\tau \ll 1\left(t_{\mathrm{mag}} \ll t_{\mathrm{th}}\right)$, rapid propagation of vortices is accompanied by adiabatic heating of the superconductor; i.e., there is not time enough to redistribute and

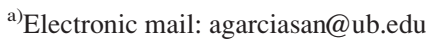

remove the heat released due to vortex motion. For $\tau \gg 1\left(t_{\text {mag }} \gg t_{\mathrm{th}}\right)$, the spatial distribution of vortices remains fixed during the stage of rapid heating. These adiabatic $(\tau \ll 1)$ and dynamic $(\tau \gg 1)$ limits are used to approach the vortex avalanche (flux jump) problem in a simplified way. ${ }^{2}$

The conventional theory of TMI considers only uniform flux jumps where the vortex front is smooth and essentially straight. ${ }^{2,4}$ Nevertheless, numerous magneto-optical imaging (MOI) studies performed in thin film superconductors made of $\mathrm{Nb}^{5,6} \mathrm{Nb}_{3} \mathrm{Sn}^{7} \mathrm{NbN}^{8} \mathrm{MgB}_{2},{ }^{9-12} \mathrm{YNiB}_{2} \mathrm{C},{ }^{13} \mathrm{~Pb},{ }^{14}$ and $\mathrm{YBa}_{2} \mathrm{Cu}_{3} \mathrm{O}_{7-\delta},{ }^{15,20}$ placed in a perpendicular dc magnetic field, have revealed that TMI can result in strongly branched dendritic flux patterns. Actually, by using MOI it has been possible to visualize the spatial distribution of the magnetic flux before and after the thermomagnetic breakdown when the magnetic field is ramped (increased or decreased) 5-8,10-14 $^{5-1}$ or when the flux jump is triggered by pulsed transport currents. ${ }^{9}$ Vortex instabilities have been also observed in the microwave (MW) response of $\mathrm{MgB}_{2}$ resonators as jumps in the resonance curves of the frequency-dependent complex transmission coefficient. ${ }^{16,17}$ Recently, vortex avalanches in $\mathrm{Pb}$ and $\mathrm{Nb}$ superconducting films, induced respectively by vortex depinning ${ }^{18}$ and ac magnetic fields, ${ }^{19}$ have been reported. In the former, the avalanche was triggered at the edge by rising the temperature locally while in the latter it appears as a result of a train of ac field cycles.

TMI has been also detected in cuprate high-temperature superconductors (HTS). ${ }^{3,4,15,20-25}$ These materials are often susceptible to flux creep, a phenomenon which can stabilize the critical state against flux jumping, ${ }^{21,22}$ making thus HTS more resistant to TMI than conventional superconductors. However, by combining MOI and femtosecond pulsed laser 
technique on films of $\mathrm{YBa}_{2} \mathrm{Cu}_{3} \mathrm{O}_{7-\delta}$, it has been possible to record a snapshot of the evolving flux distribution during the vortex avalanche. ${ }^{15,20}$ Magnetic flux jumps appear also in magnetic hysteresis loops of highly textured polycrystalline samples of $\mathrm{Bi}_{2} \mathrm{Sr}_{2} \mathrm{CaCu}_{2} \mathrm{O}_{8+\delta}$ (Refs. 23 and 24) and single crystals of $\mathrm{La}_{1.86} \mathrm{Sr}_{0.14} \mathrm{CuO}_{4}$ and $\mathrm{La}_{1.85} \mathrm{Sr}_{0.15} \mathrm{CuO}_{4}{ }^{24,25}$ The investigation of TMI in HTS is relevant to understand the complexity of the vortex matter in the mixed state of these materials. From the point of view of the applications, flux jumps are problematic as they can drive the superconductors into a normal or resistive state. On the other hand, one important drawback in interpreting the vortex avalanche phenomenon by performing conventional magnetometry is the pause time before each magnetization data point is recorded. Although during this time the magnetic field remains unchanged, the flux inside the superconductor continues evolving as a relaxation process, causing additional heating. Part of this heat is removed by the coolant while the remainder compels the temperature of the superconductor to increase, and this may lead to the occurrence of an avalanche.

In this work we present temporal resolution measurements of the voltage generated by fast vortex motion when single MW pulses are applied at fixed values of the perpendicular dc magnetic field and temperature to a superconducting thin film made of $\mathrm{La}_{2-x} \mathrm{Sr}_{x} \mathrm{CuO}_{4}(x=0.18)$. The output voltage curves of the magnetometer used to perform the experiments show a slope with a significant variation below certain threshold values of temperature, magnetic field, and pulse duration, which is interpreted as the signature of an avalanche-like vortex penetration.

\section{EXPERIMENT}

Epitaxial overdoped $\mathrm{La}_{1.82} \mathrm{Sr}_{0.18} \mathrm{CuO}_{4}$ thin films were prepared in situ by dc sputtering from stoichiometric targets on $\mathrm{SrTiO}_{3}$ substrates. The deposition chamber was first pumped to ultra high vacuum $\left(\sim 10^{-6}\right.$ mbar $)$ with the combination of a turbomolecular and a rotational pump and then filled with pure oxygen at a pressure of 2 mbar. The substrates were attached with a silver paste to a heater which was held at $\sim 840^{\circ} \mathrm{C}$ by a temperature controller and monitored with a $\mathrm{Ni} / \mathrm{NiCr}$ thermocouple. To ensure the good quality of the film, a pre-sputtering of about $1 \mathrm{~h}$ was performed at 1.9 mbar with pure oxygen and with a sputtering current held at $250 \mathrm{~mA}$ and $\sim 300 \mathrm{~V}$ over the target. Keeping the same pressure and voltage and turning the substrate in front of the target, epitaxial thin films of around $200 \mathrm{~nm}$ were obtained after about $2 \mathrm{~h}$ of sputtering. After deposition, the samples were annealed at $600^{\circ} \mathrm{C}$ in 1 bar of $\mathrm{O}_{2}$ for $1 \mathrm{~h}$ followed by slow cooling to room temperature.

A square sample with a cross-section of $3 \times 3 \mathrm{~mm}^{2}$ and a thickness of $200 \mathrm{~nm}$ was chosen to explore the flux penetration in the dynamic case, namely, by registering at low temperature the temporal variation of the magnetization when a static dc magnetic field plus a single MW pulse were applied simultaneously. Figure 1 illustrates the experimental setup. The magnetization was measured by using a commercial superconducting quantum interference device (Quantum Design rf-SQUID) magnetometer with a superconducting

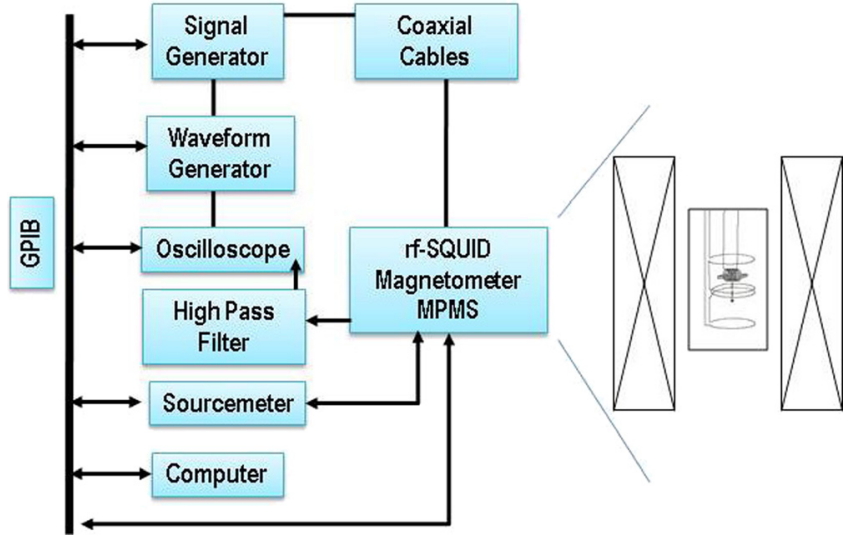

FIG. 1. Illustration of the experimental setup.

solenoid applying dc magnetic fields up to $5.5 \mathrm{~T}$ and a temperature controller with a stability better than $10 \mathrm{mK}$ at low temperature. Figure 2 plots the temperature dependence of the low-magnetic-field $(H=10 \mathrm{Oe})$ magnetization obtained in zero-field-cooling (ZFC) and field cooling (FC) modes. A superconducting transition temperature $T_{c} \sim 26 \mathrm{~K}$ is observed. In order to create a fast, controlled perturbation on the metastable critical state of the superconductor, a MW probe was designed to be used inside the magnetometer. The probe is formed by a radio-frequency (rf) coil and a 2-m-long semi-rigid stainless steel coaxial cable. The driving rf-coil was a 10-turn 1.55 -mm-long solenoid made by hand-winding a 0.1 -mm-thick, $99.9 \%$-pure copper wire on an ellipsoidal hollow plastic straw of around $11.6 \mathrm{~mm}^{2}$ cross-section. The sample, wrapped in a $25.4-\mu$ m-thick dielectric Teflon sheet, was located inside the rf-coil so that its surface ( $a b$-plane) was perpendicular to the applied dc magnetic field. The MW excitation pulse was provided to the MW probe by using an external power supply (Agilent E4433B ESG Series Signal Generator model) modulated by a function/arbitrary waveform generator (Agilent 3320A-20 MHz model).

By keeping fixed the coil-sample set in the central region of the magnetometer, the time-dependence of the output voltage signal from the rf-SQUID, $V$, was obtained with a temporal resolution of $0.1 \mathrm{~ms}$ after either a $\mathrm{ZFC}$ or a FC mode at constant MW pulse frequency $(f=0.9 \mathrm{GHz})$, at different fixed values of dc magnetic field $H$, temperature $T$,

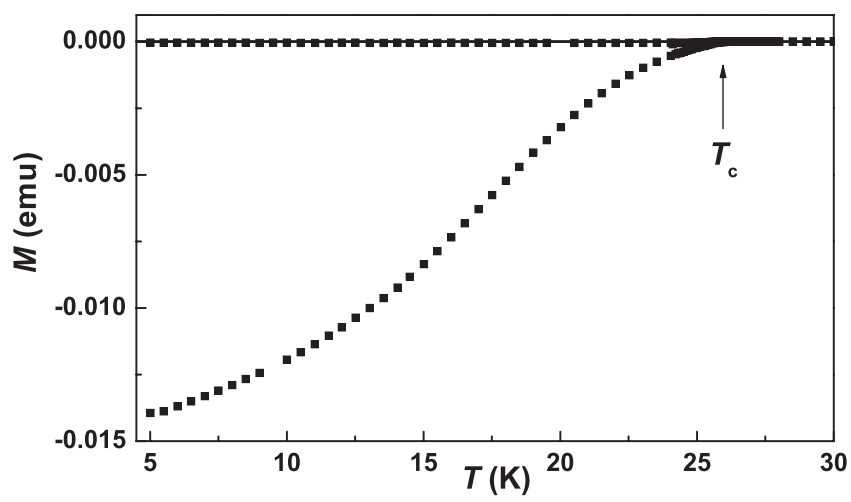

FIG. 2. Temperature dependence of the magnetization obtained at $H=10$ Oe under zero-field-cooling and field-cooling processes. 
and MW perturbation sensing parameters (pulse duration $t_{\text {pulse }}$ and nominal input power $P$ ). During these measurements the superconducting magnet was operated by an external sourcemeter (Keithley 2400 Series model). No attenuators or amplifiers were used, but due to the cable and connector losses, a reduction of $30 \mathrm{~dB}$ between the nominal applied power at the generator output $(15 \mathrm{dBm}$, that is around $30 \mathrm{~mW})$ and the incident power at the rf-coil $(-15 \mathrm{dBm}$, that is around $0.03 \mathrm{~mW}$ ), was reported at room temperature. As a consequence, MW-induced effects such as strong-driving nonlinearities or heating can be considered reduced or negligible.

The temporal variation of $V$ with respect to the first registered value which is used as a reference $\Delta V(t)$ was collected from the rf-SQUID through a high pass filter and plotted in the time domain scale of an oscilloscope (Agilent 54621A model). All measurements were controlled by a computer via a General Purpose Interface Bus (GPIB). The reproducibility was confirmed by remeasuring at different times and under the same initial conditions. No differences in $\Delta V(t)$ were observed when the MW pulse was applied after either a ZFC or a FC mode, in good agreement with earlier reports on avalanche-like flux penetration in ZFC mode in the magnetization isotherms $M(H)$ in single crystals of $\mathrm{La}_{1.85} \mathrm{Sr}_{0.15} \mathrm{CuO}_{4}$ (Refs. 3 and 22) and other superconductors ${ }^{8,11,12,23,24}$ or those performed in the remnant state by MOI. ${ }^{9,20}$ Finally, we would like to remark that the data reading capability of the pulsed MW technique used here is comparable to that of the magnetization measurements performed to detect TMI by ramping the magnetic field. ${ }^{12}$

\section{RESULTS AND DISCUSSION}

Figure 3 presents the time dependence of $\Delta V$ when MW pulses of $P=15 \mathrm{dBm}$ (around $30 \mathrm{~mW}$ ) and $t_{\text {pulse }}=15 \mathrm{~ms}$ are applied at $T=2.50 \mathrm{~K}$ for different values of $H$, from 50 to 350 Oe [panel (a)] and from 400 to 700 Oe [panel (b)]. Panels (c) and (d) show magnifications of the low-time range (0-100 ms) data of panels (a) and (b), respectively. The corresponding pulsed square wave is plotted in all panels as a reference (solid line). $\Delta V(t)$ follows different trends which extend beyond the MW pulse duration. For the lowest value of the magnetic field, $H=50$ Oe [lowermost curve in panels (a) and (c)], four sections are distinguished. In the first one, before the MW pulse application, a rather flat behavior is observed. In the second one, a deviation from the initial null value appears as a small slope, which changes to a paraboliclike response in the third section, until a maximum, named here as $\Delta V_{\text {total }}$, is reached. In the end, a plateau is achieved in the fourth section. At $H=100 \mathrm{Oe}$, five different phases [labeled 1-5 for the second curve to bottom in panel (c)] can
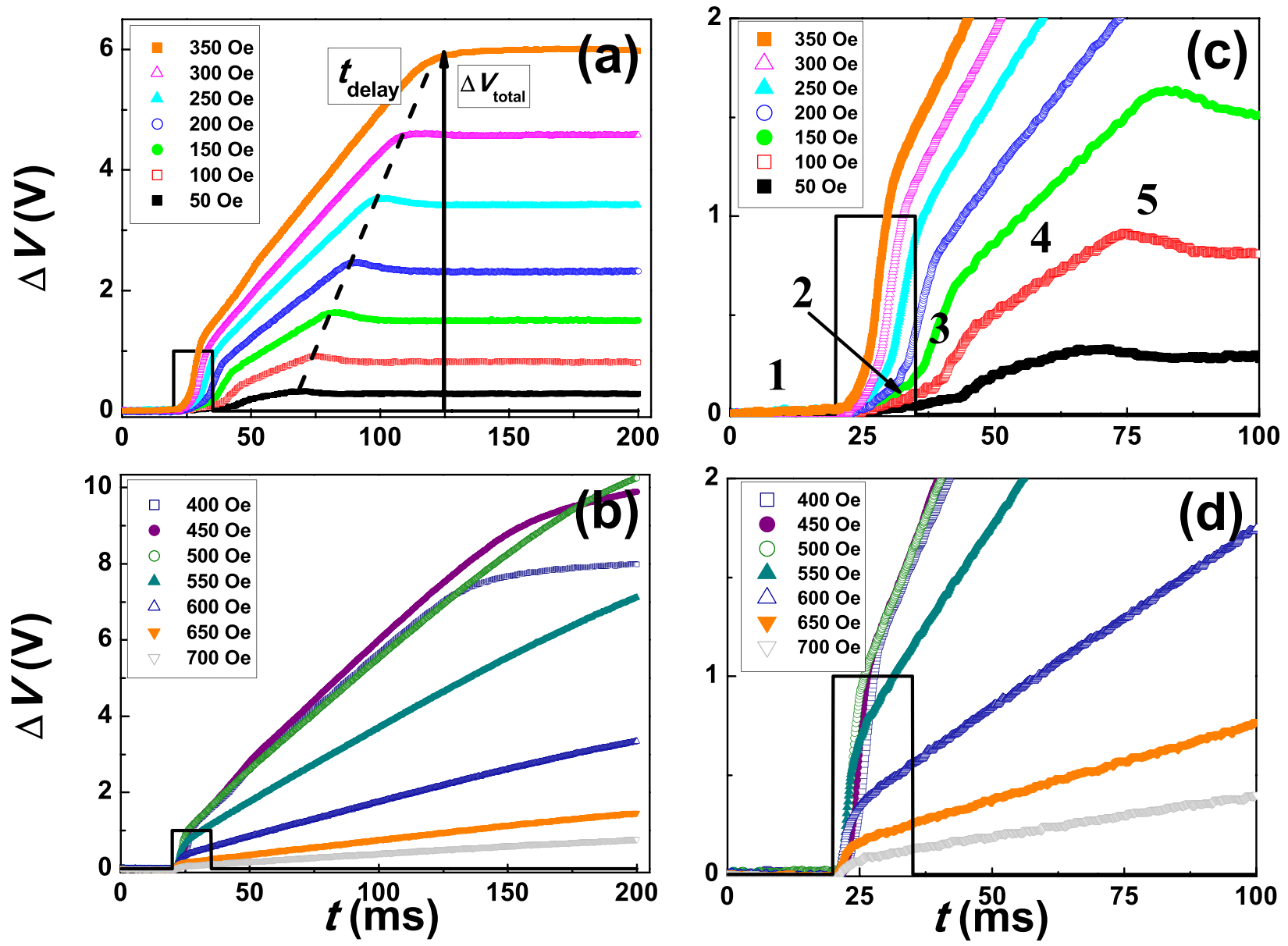

FIG. 3. Time evolution of the rf-SQUID output voltage signal variation, $\Delta V(t)$, when MW pulses of $P=15 \mathrm{dBm}$ and $t_{\text {pulse }}=15 \mathrm{~ms}$ are applied at $T=2.50 \mathrm{~K}$ for different values of $H$, from 50 to 350 Oe [panel (a)] and from 400 to 700 Oe [panel (b)]. Panels (c) and (d) show magnifications of the low-time data for panels (a) and (b), respectively (see legends for details). The corresponding pulsed square wave is shown in all panels as a reference (solid line). 
be differentiated. The initial, constant value of phase 1 is followed here in phase 2 by a more pronounced slope as compared with that described for $H=50 \mathrm{Oe}$. Then, in phase 3 the signal grows noticeably during a short time interval in a steep, jump-like way up to a certain value, from now on defined as $\Delta V_{\text {fast }}$, above which a new less conspicuous slope develops in phase 4. Finally, after $\Delta V_{\text {total }}$ as defined for $H=50$ Oe is overtaken, a smooth behavior is shown in phase 5 .

The presence of these five phases is observed in all curves as the magnetic field increases up to $H=500 \mathrm{Oe}$ although the slopes become more prominent and result in higher values for both $\Delta V_{\text {fast }}$ and $\Delta V_{\text {total }}$ [panels (c) and (d)]. Furthermore, the occurrence of $\Delta V_{\text {total }}$ is delayed in a linear way as $H$ increases [see panel (a)]. Between $H=500$ and $700 \mathrm{Oe}$ [panels (b) and (d)], $\Delta V(t)$ describes only two phases: the low-time fast one and a second stage with a slope that increases in a monotonous way and prolongs for most of the duration of the measurement. In this magnetic field regime, both $\Delta V_{\text {fast }}$ and the steepness of the curves decrease as $H$ increases. It is worth mentioning here that the existence of a small hump as $\Delta V(t)$ reaches its maximum value at the lowest magnetic fields (up to $250 \mathrm{Oe}$, approximately) is related rather with the filtering process than with the sample response.

The magnetic field dependence of the characteristic values of the output signal is depicted in logarithmic scale in Figure 4. Here, $\Delta V_{\text {total }}$ (solid squares) increases up to $H=500 \mathrm{Oe}$, where the curve reaches a maximum value of $\sim 10.26 \mathrm{~V}$ and eventually decreases rapidly down to $\sim 0.77 \mathrm{~V}$ at $H=700$ Oe. For the highest fields, since $\Delta V_{\text {total }}$ is not observed in our temporal scale, we have chosen as a reference the maximum value of $\Delta V(t)$ measured at each magnetic field. As $H$ is further enhanced, no variation in $\Delta V(t)$ is observed, evidencing that there is an upper threshold field at which the MW-pulse-induced change in the flux penetration terminates. Additionally, Figure 4 plots $\Delta V_{\text {fast }}$ (solid dots) as a function of the magnetic field. Similarly to $\Delta V_{\text {total }}, \Delta V_{\text {fast }}$ grows nearly straight from $\sim 0.14 \mathrm{~V}$ at $H=50 \mathrm{Oe}$ to $\sim 1.25 \mathrm{~V}$ at $H=500 \mathrm{Oe}$ and then drops suddenly to $\sim 0.10 \mathrm{~V}$ at $H=700$ Oe.

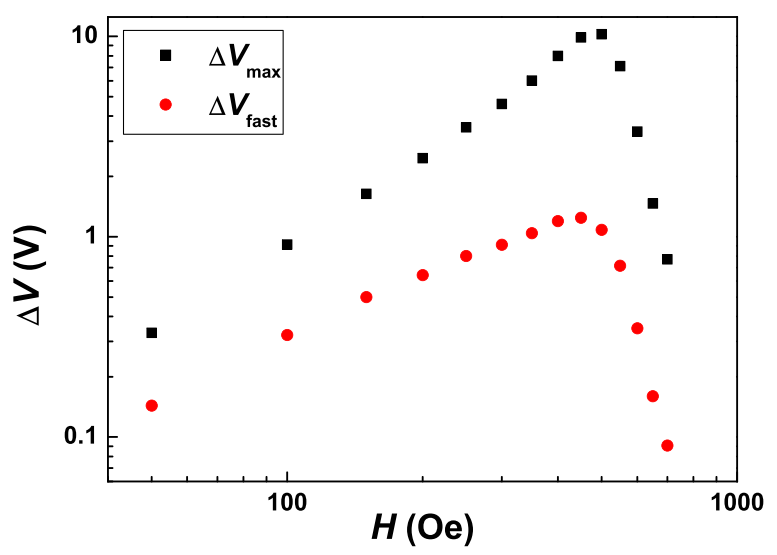

FIG. 4. Magnetic-field dependence of the fast and total rf-SQUID output voltage signal variations, $\Delta V_{\text {fast }}$ (solid dots) and $\Delta V_{\text {total }}$ (solid squares), respectively, at $T=2.50 \mathrm{~K}$ under $\mathrm{MW}$ pulses of $P=15 \mathrm{dBm}$ and $t_{\text {pulse }}=15 \mathrm{~ms}$.
Panel (a) in Figure 5 presents the time dependence of $\Delta V$ for different values of temperature, from $2.00 \mathrm{~K}$ to $6.00 \mathrm{~K}$, when MW pulses of $P=15 \mathrm{dBm}$ and $t_{\text {pulse }}=15 \mathrm{~ms}$ are applied at $H=200 \mathrm{Oe}$. At the lowest temperatures $(2.00 \mathrm{~K}$ and $2.50 \mathrm{~K})$, only a small monotonous growth, which reaches a small value of $\Delta V_{\text {total }}$ as defined in Figure 3, is distinguished after the pulse application. From $3.00 \mathrm{~K}$ to $5.00 \mathrm{~K}$, three phases can be told apart. The initial flat one before the pulse is followed by a second phase that achieves $\Delta V_{\text {fast }}$, as defined in Figure 3, with a slope that increases with temperature. After $\Delta V_{\text {total }}$ is attained, a small decrement followed by a large plateau is obtained. At $T=5.50 \mathrm{~K}$, the value of $\Delta V_{\text {fast }}$ is smaller than those shown at lower temperatures and a new phase that rises in a parabolic-like way is observed until $\Delta V_{\text {total }}$ is achieved at around $150 \mathrm{~ms}$. Finally, at $T=6.00 \mathrm{~K}$ only a flat behavior is found. The temperature dependence of $\Delta V_{\text {total }}$ (solid squares) and $\Delta V_{\text {fast }}$ (solid dots) are shown in panel (b) of this figure. $\Delta V_{\text {total }}$ increases from around $T=3.00 \mathrm{~K}$ up to a maximum at $T=5.50 \mathrm{~K}$ and then decreases rapidly and disappears at around $T=6.00 \mathrm{~K}$, indicating the existence of an upper threshold value of temperature at which the MW-pulse-induced fast flux front ceases. On the other hand, $\Delta V_{\text {fast }}$ grows as $\Delta V_{\text {total }}$ does until it reaches a maximum at around $T=4.50-5.00 \mathrm{~K}$ and then decreases in an almost linear way down to $T=6.00 \mathrm{~K}$. The filter effect on the voltage signal, as it was observed in Figure 3, appears here in the temperature range between $2.00 \mathrm{~K}$ and $4.50 \mathrm{~K}$.
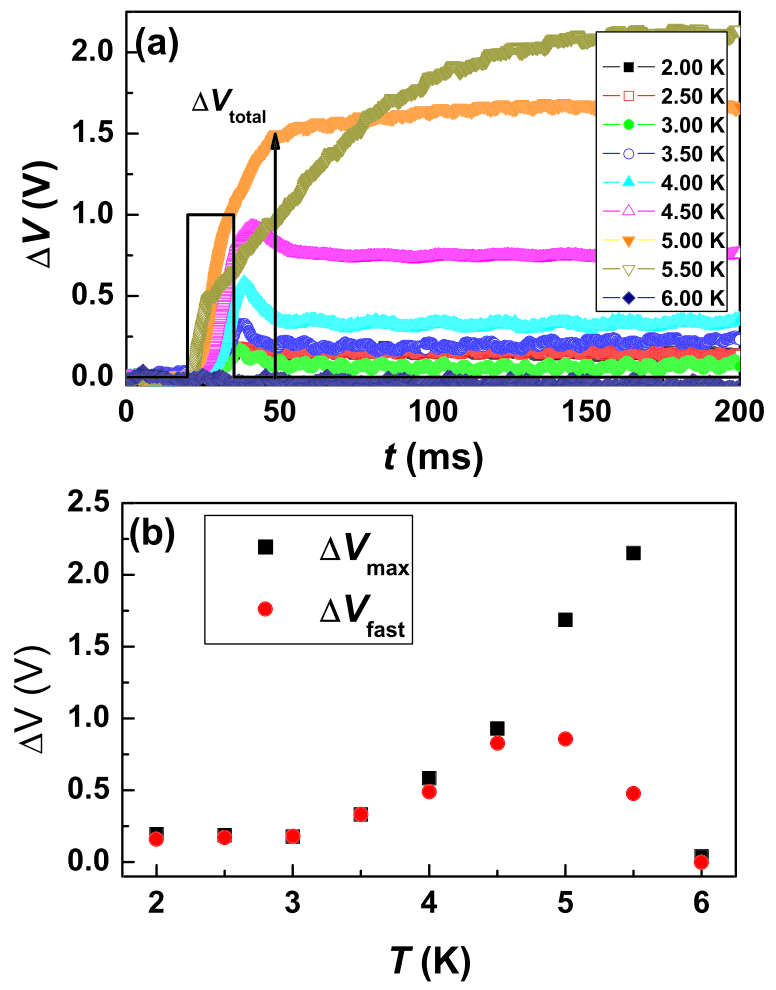

FIG. 5. (a) Temporal dependence of the rf-SQUID output voltage signal variation, $\Delta V(t)$, when MW pulses of $P=15 \mathrm{dBm}$ and $t_{\text {pulse }}=15 \mathrm{~ms}$ are applied at $H=200 \mathrm{Oe}$, for different values of temperature from $2.00 \mathrm{~K}$ to $6.00 \mathrm{~K}$ (see legend for details). The corresponding pulsed square wave is shown as a reference (solid line). (b) Temperature dependence of the fast and total rf-SQUID output voltage signal variation, $\Delta V_{\text {fast }}$ (solid dots) and $\Delta V_{\text {total }}$ (solid squares), respectively, as defined in panel (a). 
Panel (a) in Figure 6 shows the dependence of $\Delta V_{\text {total }}$ (solid squares) and $\Delta V_{\text {fast }}$ (solid dots), on $t_{\text {pulse }}$, for MW pulses of $P=15 \mathrm{dBm}$ applied at $T=2.50 \mathrm{~K}$ and $H=200 \mathrm{Oe}$. The behavior of $\Delta V(t)$ for $t_{\text {pulse }}=25 \mathrm{~ms}$, which presents the different flux penetration phases as shown in Figure 3 for different $H$ values, is plotted as an example in panel (b) of this figure. The maximum variation in the sample magnetization increases almost in a linear way with the enhancement of $t_{\text {pulse }}$ from $\sim 3 \mathrm{~ms}$ to $\sim 40 \mathrm{~ms}$ and then starts to decline and finishes at around $60 \mathrm{~ms}$, demonstrating that the MW-pulseinduced instability depends also on the duration of the pulse. On the other hand, $\Delta V_{\text {fast }}$ [phase 3 in panel (b)] reaches a maximum slightly before $10 \mathrm{~ms}$ and then remains almost constant up to about $35 \mathrm{~ms}$, when it starts to decrease slowly and finally vanishes at around $60 \mathrm{~ms}$. As it is observed in panel (b), $\Delta V_{\text {fast }}$ is followed by a long relaxation process until a maximum in the voltage variation is achieved.

Panel (a) in Figure 7 plots the time dependence of $\Delta V(t)$ for different values of nominal power, from $9 \mathrm{dBm}$ (circa $8 \mathrm{~mW}$, lowermost curve) to $19 \mathrm{dBm}$ (circa $80 \mathrm{~mW}$, uppermost curve) in steps of $0.5 \mathrm{dBm}$, when MW pulses of $t_{\text {pulse }}=15 \mathrm{~ms}$ are applied at $H=500 \mathrm{Oe}$ and $T=2.50 \mathrm{~K}$. The corresponding pulsed square wave is plotted as a reference (dashed line). The behavior of the $\Delta V(t)$ curves is similar, in general, to that shown in panel (a) of Figure 3: a flat initial phase previous to the pulse is followed by a smooth slope, after which a jump-like phase takes place until a new stage with a reduced slope appears, and a maximum that leads to a
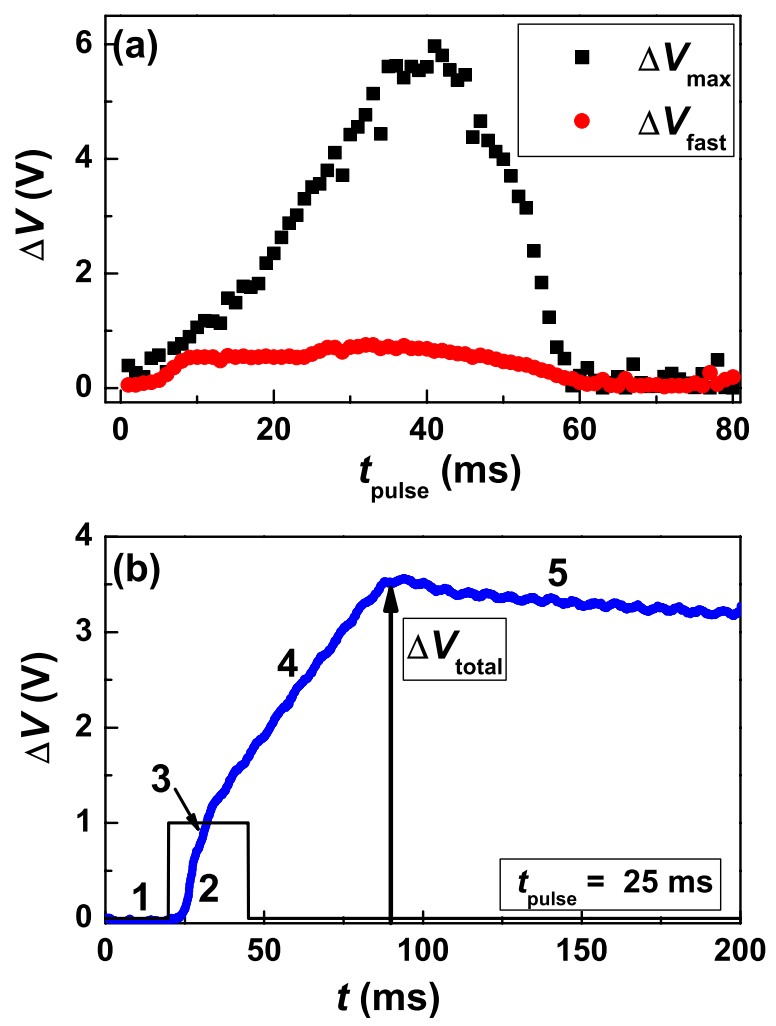

FIG. 6. (a) Pulse duration dependence of the fast and total rf-SQUID output voltage signal variation, $\Delta V_{\text {fast }}$ (solid dots) and $\Delta V_{\text {total }}$ (solid squares), respectively, for MW pulses of $P=15 \mathrm{dBm}$, at $T=2.50 \mathrm{~K}$ and $H=200 \mathrm{Oe}$. (b) Temporal dependence of the voltage signal variation, $\Delta V(t)$, generated at $t_{\text {pulse }}=25 \mathrm{~ms}$. The corresponding pulsed square wave is shown as a reference (solid line).
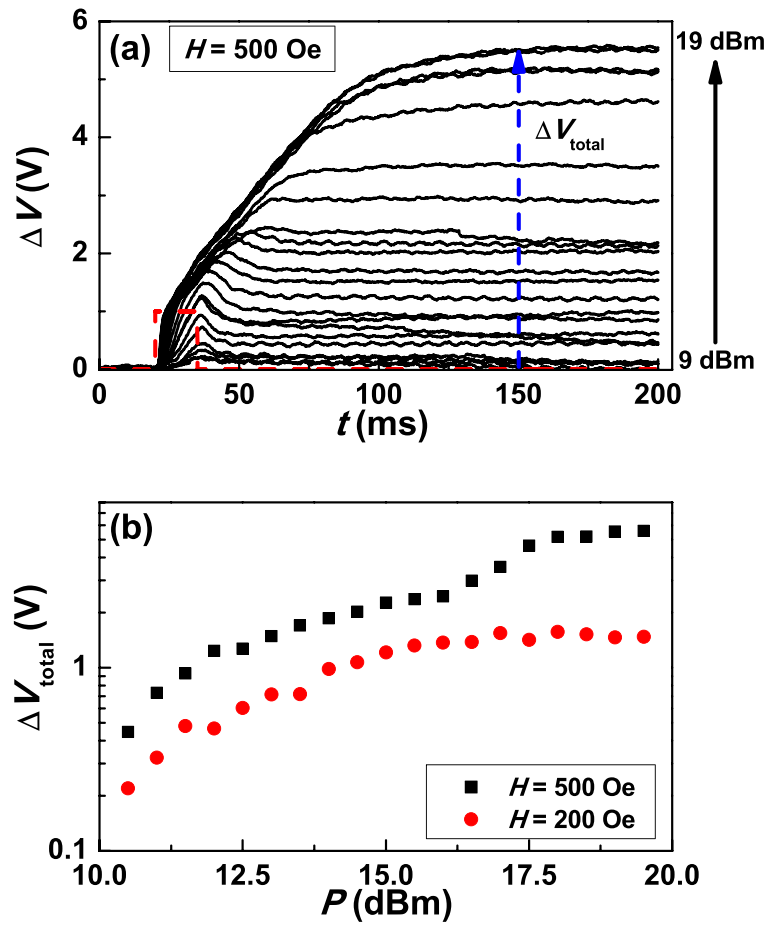

FIG. 7. (a) Temporal dependence of the rf-SQUID output voltage signal variation, $\Delta V(t)$, when MW pulses of $t_{\text {pulse }}=15 \mathrm{~ms}$ and different nominal powers, from $9 \mathrm{dBm}$ (lowermost curve) to $19 \mathrm{dBm}$ (uppermost curve), are applied at $H=500 \mathrm{Oe}$ and $T=2.50 \mathrm{~K}$. (b) Nominal power dependence of the total rf-SQUID output voltage signal variation as defined in panel (a), $\Delta V_{\text {total }}$, for $H=200 \mathrm{Oe}$ (solid dots) and $H=500 \mathrm{Oe}$ (solid squares).

final plateau is reached. In this plot, $\Delta V_{\text {total }}$ appears to be completed relatively earlier with the enhancement of $P$ for the lowest powers up to around $P=10 \mathrm{dBm}(10 \mathrm{~mW})$. Above this power value, $\Delta V_{\text {total }}$ is fulfilled with a considerable delay. Here, the effect of the filter on the voltage response as it was defined in Figure 3 is significant for $P \simeq 10.5-15.5 \mathrm{dBm}$ (around 11-35 W). Panel (b) in this figure shows in semilogarithmic scale the nominal power dependence of $\Delta V_{\text {total }}$ as defined in panel (a), for $H=200 \mathrm{Oe}$ (solid dots) and $H=500$ Oe (solid squares). In accordance with the results obtained in Figure 3 for $P=15 \mathrm{dBm}$, now the value of $\Delta V_{\text {total }}$ for $H=500 \mathrm{Oe}$ is larger than that for $H=200 \mathrm{Oe}$ at any $P$ value. In both cases, as $P$ increases, $\Delta V_{\text {total }}$ grows to achieve a maximum around $P=15-16 \mathrm{dBm}$ (circa $32-40 \mathrm{~mW}$ ), after which it stays almost constant for $H=200 \mathrm{Oe}$, whereas it shows a short growth until a new smooth regime appears for $H=500$ Oe. Since the power received by the sample is reduced in $30 \mathrm{~dB}$ with respect to the input nominal power by the losses in the cable and connectors, it seems reasonable that the MW-pulse-induced fast flux movement does not appear until a significant value of $P$ is realized.

Since $\Delta V(t)$ actually reflects the temporal variation of the sample magnetization, we can associate the MW-pulseinduced voltage signal with the time-resolved behavior of the flux penetration which occurs in different phases. Thus the smooth phase before the pulse is applied can be ascribed in all figures shown to a slow and steady vortex diffusion which follows the Bean-like profile. By applying a MW pulse, the initial profile is perturbed so that a rapid flux front is induced and a new phase appears. As described in the 
introduction, this flux motion increases the local temperature, reducing therefore the critical current density and triggering further vortex motion. This feedback produces an enhancement of the amount of vortices that enter the sample rapidly and leads to the achievement of $\Delta V_{\text {fast }}$. This $\mathrm{MW}$-assisted flux penetration is extended in a subsequent phase, but this happens at reduced velocity as the vortices move through the sample and finally ceases at $\Delta V_{\text {total }}$. Two different effects, namely, intrinsic pinning and heat transfer from the film to the substrate and the coolant, could be behind the reduction in the vortex propagation. Moreover, we cannot rule out the contribution that may come from the flux creep effect in this rather long phase and the delay to reach $\Delta V_{\text {total }}$ as the magnetic field [see Figure 3(a)], the temperature [see Figure 5(a)], or the power increase [see Figure 7(a)]. As a matter of fact, the flux creep would change the flux profile inside the sample and thus the shape of $\Delta V(t)$. Finally, the flat response observed in the last phase could be referred either to gradual vortex propagation, with a slower dynamics with respect to the fast flux penetration, or to the existence of a magnetic field profile which remains frozen once the flux stops spreading. More information about this apparently smooth magnetic variation should be obtained from MOI, where the spatial distribution of vortices at this stage could be captured with high time resolution.

Our measurements show that the slopes of the different sections in the $\Delta V(t)$ curves change with the magnetic field (Figure 3), the temperature [Figure 5(a)], the pulse duration [Figure 6(b)], and the power [Figure 7(a)]. As $H$ is enhanced from $50 \mathrm{Oe}$ up to $500 \mathrm{Oe}$, the slopes get more pronounced, and $\Delta V_{\text {total }}$ increases suggesting that a higher density of vortices is driven from the edge towards the center of the sample with the rise of the magnetic field. The reduction of $\Delta V_{\text {fast }}$ and consequently of the slope of $\Delta V(t)$ above $H=500 \mathrm{Oe}$ implies that the MW-pulse-assisted flux motion becomes less effective at these intensities. Similarly, as $T$ increases from $3.00 \mathrm{~K}$ to $5.00 \mathrm{~K}$, the slopes become significant meaning that there is a range of intermediate temperatures at which the fast vortex motion could be MW-pulse-assisted. A comparable behavior has been also registered for the $\Delta V(t)$ curves acquired for values of $t_{\text {pulse }}$ between $10 \mathrm{~ms}$ and $45 \mathrm{~ms}$. Not surprisingly, the range of fast flux occurrence appears conditioned by the duration of the pulse since as $t_{\text {pulse }}$ increases, so it does the excitation time that is needed to maintain this assisted motion. Nevertheless, the existence of a maximum value in $t_{\text {pulse }}$ suggests that once it is reached, a further enhancement of the pulse duration will imply a longer time for the sample to transfer the heat to the environment giving as a consequence a reduction in the rapid flux penetration. We cannot rule out either the contribution to this diminution that may come from the relaxation of the magnetic moment of the sample.

The existence of recurrent signatures of fast flux motion in the $\Delta V(t)$ curves as a function of $T, H, t_{\text {pulse }}$, and $P$, and below certain values of all these magnitudes but the nominal power allows us to attribute the jump-like behavior in the variation of the magnetization of our sample to the nucleation of a TMI induced by the single MW pulse. In order to shed light on the origin of such MW-pulse-induced fast flux diffusion we will consider the possible contribution to the development of TMI from the electric and magnetic MW fields, $E_{\mathrm{MW}}$ and $H_{\mathrm{MW}}$, respectively, created at the edge of the thin film by the rf-coil when the MW pulse is applied. We have estimated $E_{\mathrm{MW}} \simeq 34 \mathrm{~V} / \mathrm{m}$ and $H_{\mathrm{MW}} \simeq 2.0 \mathrm{mOe}$, by using the rf-coil approximation ${ }^{26}$ and the following experimental data: $f=0.9 \mathrm{GHz}, \varepsilon_{r}=300$ for the dielectric constant of the $\mathrm{SrTiO}_{3}$ substrate, $P=0.01 \mathrm{~mW}(-20 \mathrm{dBm})$ for the lower effective incident power at the rf-coil, and $Q=100$ for the rf-coil quality factor. This calculation, to be considered elsewhere, provides arguments to suggest the mechanism that facilitates the sudden jump in $\Delta V(t)$. In addition to its very low value, the lack of uniformity of $H_{\mathrm{MW}}$ due to the limited length of the coil with respect to the sample size enables us to ignore its contribution. On the contrary, the value obtained for $E_{\mathrm{Mw}}$ (which is much higher than the electric field of $0.01 \mathrm{~V} / \mathrm{m}$ obtained at full flux penetration in the Bean model ${ }^{27}$ ) is relevant for the ignition of the flux bursts. In fact, a value of around $1 \mathrm{~V} / \mathrm{m}$ has been reported for the maximum electric field during the ignition of avalanche-like behavior. ${ }^{28}$ Therefore, we can state that there is an additional electric field originated by the MW pulse at the edge of the sample that contributes considerably to the existing background electric field making the latter to increase enough to favor avalanche-like vortex penetration. The contribution of the background field as a source for triggering the vortex instability has been confirmed theoretically ${ }^{29}$ and experimentally. ${ }^{12,15,20}$ One important consequence of this argument is that the adiabatic approach $(\tau \ll 1)$ that supports the appearance of flux jumps may be fulfilled since $\tau$ is inversely proportional to the flux flow resistivity, which in turn increases as the electric field does.

\section{CONCLUSIONS}

We have studied the temporal evolution of the flux penetration in an epitaxial overdoped $\mathrm{La}_{1.82} \mathrm{Sr}_{0.18} \mathrm{CuO}_{4}$ thin film when single MW pulses are applied as a perturbation source, by registering the output voltage signal from an rf-SQUID magnetometer. MW-pulse-induced fast vortex penetration is observed in the form of a jump-like behavior that occurs while the MW pulse is applied below threshold values of dc magnetic field, temperature and pulse duration. The evolution of the shape and height of the output signal could be influenced by flux creep phenomena and heat transfer, particularly in the last phase of the dynamical process when the vortex motion slows down considerably and ends up freezing. The induced vortex instability is believed to be of electric field origin, similarly to the flux jumping induced by the background electric field predicted and observed during magnetic-field-swept or laser pulse measurements. The reduction of heating effects, the high sensitivity in the measurements under different thermodynamics and MW parameters, and the study of the sample response in the time scale of milliseconds are the most important advantages of the method described. Finally, a complete interpretation of our results in terms of a thermomagnetic model could be achieved by combining the technique used here with magneto-optical imaging experiments. 


\section{ACKNOWLEDGMENTS}

P.-de-J.C.-S. would like to express gratitude to A. Fernández for useful technical discussion on microwave issues. J.M.H. acknowledges support from Spanish Ministerio de Educación y Ciencia and Universitat de Barcelona. A.G.-S. acknowledges support from Universitat de Barcelona. J.T. acknowledges support from Generalitat de Catalunya ICREA Acadèmia programme. This work was financially supported by the Spanish Government Projects (Nos. MAT2008-04535 and MAT2011-23698).

${ }^{1}$ Ch. P. Bean, Phys. Rev. Lett. 8, 250 (1962).

${ }^{2}$ R. G. Mints and A. L. Rakhmanov, Rev. Mod. Phys. 53, 551 (1981).

${ }^{3}$ V. V. Chabanenko, A. I. D'yachenko, A. V. Chabanenko, H. Szymczak, S. Piechota, A. Nabiałek, and N. D. Dung, J. Appl. Phys. 83, 7324 (1998).

${ }^{4}$ S. L. Wipf, Cryogenics 31, 936 (1991).

${ }^{5}$ C. A. Durán, P. L. Gammel, R. E. Miller, and D. J. Bishop, Phys. Rev. B 52, 75 (1995)

${ }^{6}$ M. S. Welling, R. J. Westerwaal, W. Lohstroh, and R. J. Wijngaarden, Physica C 411, 11 (2004).

${ }^{7}$ I. A. Rudnev, S. V. Antonenko, D. V. Shantsev, T. H. Johansen, and A. E. Primenko, Cryogenics 43, 663 (2003)

${ }^{8}$ I. A. Rudnev, D. V. Shantsev, T. H. Johansen, and A. E. Primenko, Appl. Phys. Lett. 87, 042502 (2005).

${ }^{9}$ A. V. Bobyl, D. V. Shantsev, T. H. Johansen, W. N. Kang, H. J. Kim, E. M. Choi, and S. I. Lee, Appl. Phys. Lett. 80, 4588 (2002).

${ }^{10}$ D. V. Shantsev, P. E. Goa, F. L. Barkov, T. H. Johansen, W. N. Kang, and S. I. Lee, Supercond. Sci. Technol. 16, 566 (2003).

${ }^{11}$ E. M. Choi, H.-S. Lee, H. J. Kim, B. Kang, S.-I. Lee, A. A. F. Olsen, D. V. Shantsev, and T. H. Johansen, Appl. Phys. Lett. 87, 152501 (2005).
${ }^{12}$ Z. W. Zhao, S. L. Li, Y. M. Ni, H. P. Yang, Z. Y. Liu, H. H. Wen, W. N. Kang, H. J. Kim, E. M. Choi, and S. I. Lee, Phys. Rev. B 65, 064512 (2002).

${ }^{13}$ S. C. Wimbush, B. Holzapfel, and Ch. Jooss, J. Appl. Phys. 96, 3589 (2004).

${ }^{14}$ M. Menghini, R. J. Wijngaarden, A. V. Silhanek, S. Raedts, and V. V. Moshchalkov, Phys. Rev. B 71, 104506 (2005).

${ }^{15}$ P. Leiderer, J. Boneberg, P. Brüll, V. Bujok, and S. Herminghaus, Phys. Rev. Lett. 71, 2646 (1993).

${ }^{16}$ G. Ghigo, R. Gerbaldo, L. Gozzelino, F. Laviano, G. Lopardo, E. Monticone, C. Portesi, and E. Mezzetti, Appl. Phys. Lett. 94, 052505 (2009).

${ }^{17}$ G. Ghigo, F. Laviano, L. Gozzelino, R. Gerbaldo, E. Mezzetti, E. Monticone, and C. Portesi, J. Appl. Phys. 102, 113901 (2007).

${ }^{18}$ A. A. Awad, F. G. Aliev, G. W. Ataklti, A. Silhanek, V. V. Moshchalkov, Y. M. Galperin, and V. Vinokur, Phys. Rev. B 84, 224511 (2011).

${ }^{19}$ M. Motta, F. Colauto, T. H. Johansen, R. B. Dinner, M. G. Blamire, G. W. Ataklti, V. V. Moshchalkov, A. V. Silhanek, and W. A. Ortiz, Physica C 479, 134 (2012).

${ }^{20}$ U. Bolz, B. Biehler, D. Schmidt, B.-U. Runge, and P. Leiderer, Europhys. Lett. 64, 517 (2003)

${ }^{21}$ R. G. Mints, Phys. Rev. B 53, 12311 (1996).

${ }^{22}$ M. E. McHenry, H. S. Lessure, M. P. Maley, J. Y. Coulter, I. Tanaka, and H. Kojima, Physica C 190, 403 (1992).

${ }^{23}$ A. Nabiałek, M. Niewczas, H. Dabkowska, A. Dabkowski, J. P. Castellan, and B. D. Gaulin, Phys. Rev. B 67, 024518 (2003).

${ }^{24}$ A. Nabiałek and M. Niewczas, Physica C 436, 43 (2006).

${ }^{25}$ A. Nabiałek, A. Wisniewski, V. V. Chabanenko, S. V. Vasiliev, T. V. Tsvetkov, and F. Pérez-Rodríguez, Supercond. Sci. Technol. 25, 035005 (2012).

${ }^{26}$ K. L. Corum and J. F. Corum, Microwave Rev. 7, 36 (2001).

${ }^{27}$ E. H. Brandt, Phys. Rev. B 55, 14513 (1997).

${ }^{28}$ J. I. Vestgården, D. V. Shantsev, Y. M. Galperin, and T. H. Johansen, Sci. Rep. 2, 886 (2012).

${ }^{29}$ D. V. Denisov, A. L. Rakhmanov, D. V. Shantsev, Y. M. Galperin, and T. H. Johansen, Phys. Rev. B 73, 014512 (2006). 